\title{
Cyber-Physical Systems for Micro-/Nano-assembly Operations: a Survey
}

\author{
Jose A Mulet Alberola ${ }^{1}$ (D) Irene Fassi $^{1}$ \\ Accepted: 21 December 2020 / Published online: 21 January 2021 \\ (C) The Author(s) 2021
}

\begin{abstract}
Purpose of Review Latest requirements of the global market force manufacturing systems to a change for a new production paradigm (Industry 4.0). Cyber-Physical Systems (CPS) appear as a solution to be deployed in different manufacturing fields, especially those with high added value and technological complexity, high product variants, and short time to market. In this sense, this paper aims at reviewing the introduction level of CPS technologies in micro/nano-manufacturing and how these technologies could cope with these challenging manufacturing requirements.

Recent Findings The introduction of CPS is still in its infancy on many industrial applications, but it actually demonstrates its potential to support future manufacturing paradigm. However, only few research works in micro/nano-manufacturing considered CPS frameworks, since the concept barely appeared a decade ago.

Summary Some contributions have revealed the potential of CPS technologies to improve manufacturing performance which may be scaled to the micro/nano-manufacturing. IoT-based frameworks with VR/AR technologies allow distributed and collaborative systems, or agent-based architectures with advance algorithm implementations that improve the flexibility and performance of micro-/nano-assembly operations. Future research of CPS in micro-/nano-assembly operations should be followed by more studies of its technical deployment showing its implications under other perspectives, i.e. sustainable, economic, and social point of views, to take full advance of all its features.
\end{abstract}

Keywords Cyber-Physical Systems (CPS) - Cyber-Physical Production Systems (CPPS) - Micro-manufacturing . Nano-manufacturing $\cdot$ Micro-assembly

\section{Introduction}

Global manufacturing is currently challenged by market trends of a more personalized production demand [1] while decreasing product's life-cycle. These market requirements force to rethink manufacturing under a holistic vision as it is nowadays, and drive a change to a new manufacturing

This article belongs to the Topical Collection on Nanorobotics and Microrobotics

Jose A Mulet Alberola

jose.alberola@stiima.cnr.it

Irene Fassi

Irene.Fassi@stiima.cnr.it

1 Institute of Intelligent Industrial Technologies and Systems for Advanced Manufacturing (STIIMA), National Research Council (CNR), 20133 Milan, Italy paradigm which materializes in the Industry 4.0 (I4.0) concept [2]. This new smart manufacturing in I4.0 is supported by the adoption of adaptable, autonomous, evolvable, and collaborative systems [3] [4] [5] and founded by the introduction of the Cyber-Physical Systems (CPS) concept [6], or Cyber-Physical Production Systems (CPPS) [7] when talking about its specific application to manufacturing industry. Both concepts arise from the combination of computational and physical resources in systems. These systems are computational entities highly connected to their physical counterpart and, subsequently, rely on the latest developments on manufacturing science and technology, as well as on computer science, information, and communication technologies to provide and use dataaccessing and data-processing services simultaneously. This multi-disciplinary approach is seen with huge expectation as an extremely important step in the development of future manufacturing systems.

Some industries, as the micro/nano-manufacturing, are more affected by the aforementioned challenges, where short 
time horizons, high product variant turnovers, and semimanual assembly operations are common [8] [9] [10]. Micro/nano-manufacturing is, in general, a very specialized and sophisticated production due to the technical requirements of the scale. It defines a network of specialized and cooperative factories and enterprises involved along the whole product life-cycle, physically distributed in different teams, sharing product information, and knowledge along the cycle. The fastemerging micro-manufacturing domain is estimated to be valued $€ 64.6$ billion in 2019 and expected to reach $€ 141.6$ billion by 2024, at a CAGR of $16.9 \%$ from 2019 to 2024 [11]. The evolution of the micro/nano-manufacturing started in the 1990s when the assistance of an experienced operator was required to the use of micro-techniques combined with computer performances applied to the control equipment and vision systems to operate at a micro-scale [12]. One of the main bottlenecks is still represented by the assembly phase, which often amounts to the largest part of the final product costs. Robotic micro-/nano-assembly manufacturing faces with high complexity [13] mainly caused by the high accuracy required over a large range of motion and the complexity of reduced scale of actuators and sensors.

In order to achieve an efficient and accurate manipulation of micro-/nano-components, modelling the physics at the micro-scale plays a major role [14]. Nevertheless, significant differences are present when comparing the macro- and the micro/nano-scale behaviours [13] [15] [16], where superficial forces predominate over volumetric ones. During the last decades, basic models have been developed, although the current behaviour of the micro-process is impossible to predict due to the high sensitivity of the predominant forces to external or environmental parameters (humidity, temperature, ionic concentration in liquid...). Furthermore, micro-manipulating systems are limited in mechanical arrangements and actuation caused by the scale and traditionally require severe maintenance and complex control procedures [17]. Therefore, automated micro-/nano-assembly systems are typically very expensive, aggravated by a reduced throughput.

In response to these specific requirements, CPPS concepts represent a powerful instrument to boost micro/ nano-manufacturing. More intelligent systems based on innovative information-centric frameworks [18] and advanced algorithms may analyze and select optimized assembly operations and tools that are simulated in virtual environments allowing faster setups for evolvable, reconfigurable, and more flexible manufacturing system. The digital-twin (DT) [19] concept is a virtual representation of a physical system and is composed by multimodel simulations for real-time prediction, optimization, monitoring, and control. DT stands for an effective tool to cope with undesirable and unpredictable behaviours in systems with high complexities [20]. Collaborative operations with human operators [21] [22] will be enabled for specific assembly operations [23], combining the advantages of both actors (humans and robots) and reaching higher efficiencies by taking advance of virtual reality (VR) and augmented reality (AR) technologies. On the other hand, the Industrial Internet of Things (IIoT) [24] promises multiple devices connected to the Internet and aims at integrating operational data to the cyber system to achieve smart behaviours. The introduction of software agents and multi-agent systems (MAS) in manufacturing [25] [26] allows the deployment of these complex systems aiming at autonomy and modularity features in control activities, since agents represent fundamental processing units with advance computational capabilities. These context-aware systems will allow manufacturing systems to behave in a more autonomous manner with self-x capabilities (i.e. self-configuration, self-organization, self-learning).

This work reviews the main solutions available nowadays to implement a Cyber-Physical Production System for the micro-/nano-assembly of devices. "Cyber-Physical Technologies Towards Micro-/Nano-assembly" exposes the main solutions and methods to deploy a CPPS in the micro/ nano-manufacturing context; then, "Conclusions" draws conclusions and some considerations regarding the CPS inclusion level in this industrial field, the research work done, and future steps.

\section{Cyber-Physical Technologies Towards Micro-/Nano-assembly}

Figure 1 shows a conceptual scheme of a CPPS applied to micro-/nano-assembly operations: micro/nanocomponents (i) are manipulated following the most satisfactory strategy computed by using different models (ii). The whole sequence is controlled and optimized by the system (iii) to assembly the final micro/nanodevice (iv). Information and raw data, from either the physical world or historical database, are available for the use along the whole process (v). Human operators may contribute to the system in how to act or by validating the whole manipulation and assembly operations (vi).

The deployment of CPPS is accomplished by the use of key enabling technologies and methods from an applicationdriven analysis, which sometimes are difficult to establish or materialize in a physical system. For that reason, these enablers have been classified according to their different deployment levels in a micro-/nano-assembly application:

A. Sensing methodologies for real-time data collection by using advanced vision-based systems and sensors. 


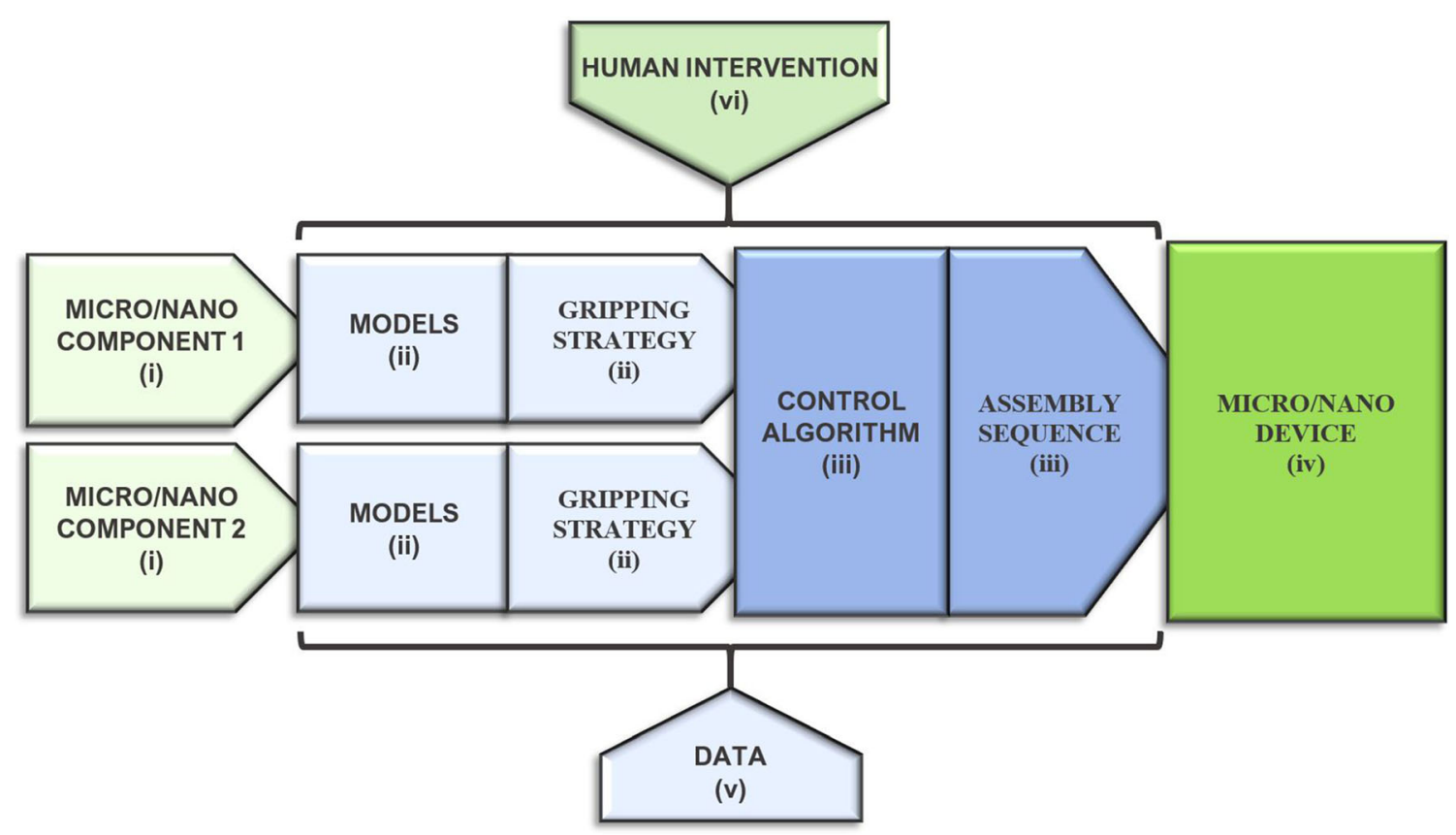

Fig. 1 Premises of using Cyber-Physical Production Systems (CPPS) in micro-/nano-assembly operations, by the combination of physical (green) and computational (blue) resources

B. Models and algorithms for autonomous gripping include physical models and algorithms for autonomous micro/nano-object detection and gripping. Sophisticated calibration methodologies are also required to meet highaccuracy requirements of vision-based systems.

C. Autonomous assembly operations based on vision tracking systems, complex assembly sequences, models, and algorithms using multi-robot collaborations.

D. Human role in assembly and human-robot interactions. Human intervention is boosted using VR/AR systems for teleoperating micro-/nano-assembly systems, direct collaboration and cooperation in assembly tasks between human operators and robots with the inclusion of distributed teams using Internet connections.

E. Advanced frameworks and control architectures based on Evolvable Production Systems (EPC), digital twins (DT), and multi-agent systems (MAS) comprehend all aforementioned methods and technologies, allowing algorithm executions for real-time analysis reaching intelligent behaviours (self-configuration, self-organization, self-diagnostic, self-learning behaviours).

Figure 2 represents how the abovementioned concepts can be actually deployed in a robotic cell for micro-assembly manufacturing. The collaborative work cell, inspired by the work presented in [27], is composed by a precision manipulator and a collaborative robot, guided by different vision systems and controlled by a digital-twin framework.

The following sections describe in more detail each category, focusing on the research works found enabling each of the abovementioned aspects of CPPS for micro-/nano-assembly operations.

\section{Sensing Methodologies for Data Collection}

Real-time sensing and processing of physical data has a major role in CPS systems, especially for an accurate and effective execution of the digital twin and a correct decision-making. Due to the care required during micro-manipulation, there is an extreme necessity of developing accurate and reliable methods for measuring forces at the micro-scale [28], which represent a very complex process as a consequence of the difficulty to fabricate and calibrate physical sensors. Additionally, force sensing at the micro-scale represents a challenge in terms of sensor resolution, sensitivity, range of work, etc. Therefore, vision systems are used to force measurements despite its challenging requirements in terms of resolution, magnification, limited field-of-view, and depth of field. Among the approaches developed, the most common are [28] as follows: (i) strain gauge-based; (ii) piezoresistive; (iii) capacitive; (iv) piezoelectric; (v) piezomagnetic; (vi) optical; (vii) vision-based.

Vision-based methods are widely used also for indirect feedback by comparison of the deformation under the applied loads and the physical model. For example, in [29], a vision system is presented for real-time force feedback providing sub- $\mu \mathrm{N}$ level force resolution in three dimensions. In [30], the control of a mobile manipulation micro-robot is achieved exploiting vision-based force sensing capabilities in the range $0-20 \mu \mathrm{N}$ and with a resolution of $1.5 \mu \mathrm{N}$ in real time. On the 
PHYSICAL WORLD

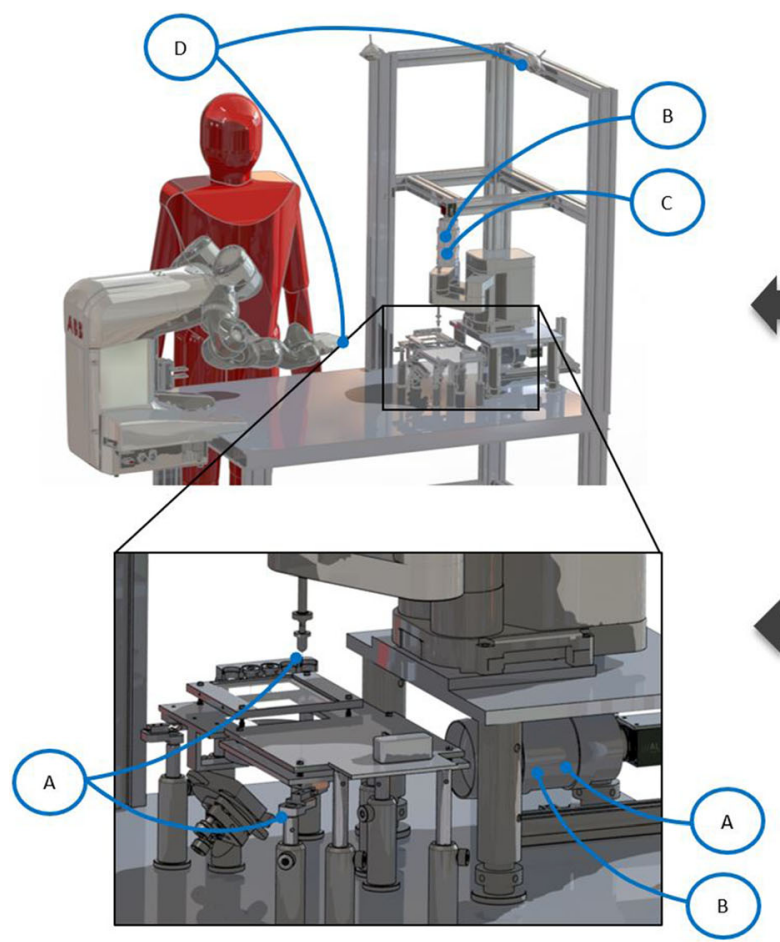

Fig. 2 Deployment of a Cyber-Physical Production System (CPPS) for micro-/nano-assembly operations. The diagram represents the physical deployment and its cyber counterpart: vision-based and sensing-based methodologies to measure forces (A), advanced gripping strategies by
CYBER WORLD

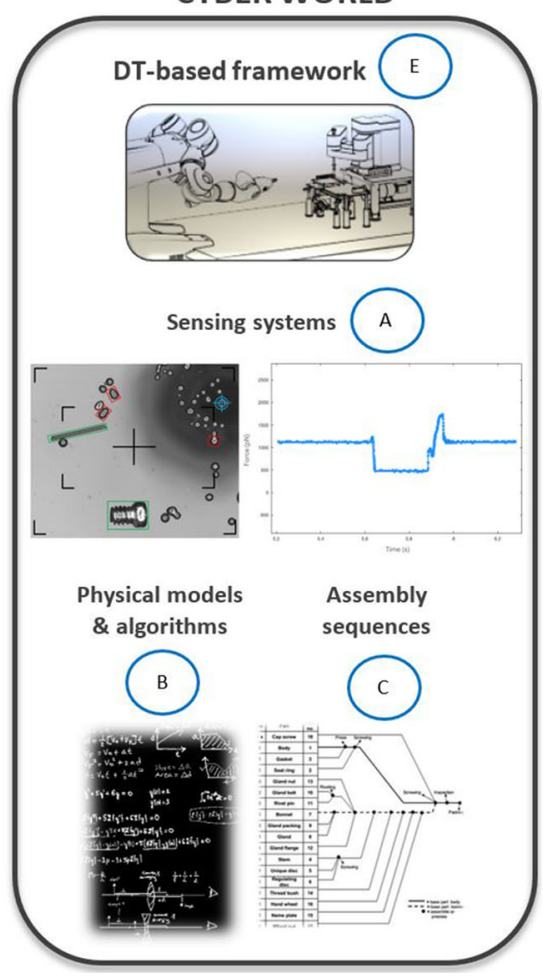

using vision recognition, physical models, and algorithms (B), assembly sequences and algorithms based on shape recognition and 3D models (C), VR/AR teleoperating systems and human-robot collaborative systems (D), and advanced frameworks and control architectures (E) other hand, in [31], a piezoelectric-driven micro-gripper is equipped with strain gauges capable of measuring either applied micro-forces or displacements by simulating its behaviour with finite elements methods. In [32], bimorph piezoelectric strips help in manipulation tasks, and force is measured by first characterizing the behaviour and the deflection of the tweezer's fingers.

\section{Models and Algorithms Towards Adaptable Gripping Operations}

The complexity of gripping operations lies in the high variety of components to be assembled and the high accuracy and fineness required. To face with these issues, not only an accurate physical system is required, but also a reliable and adaptable cyber system based on the analysis of the several different gripping strategies and physical models.

In order to achieve robustness during the manipulation (gripping or release phase) of micro/nano-objects, it is important to understand the physical interactions between the object and the gripper. Early works theoretically modelled microforce interactions (and specifically impact and contact forces) to simulate them in virtual environments [33]. Other strategies use compliant micro-grippers to avoid damages of micro- components [32] or developed specific designs based on constant force gripping mechanisms [34].

Later researches introduced vision recognition of micro/ nano-components and learning algorithms applied to recognition and tracking of micro-objects [35] presents a complete review on learning-based approaches to perform general gripping operations at the macro-scale. To enhance dexterity and optimize the grasping trajectories, authors in [36] [37•] exploited physical models of grasping forces and pull-off forces during micro-manipulation operations using search algorithms for a real-time application which computes the gripping trajectory in less than $0.1 \mathrm{~s}$. van Vuuren JJ et al. [38] propose a learning-based methodology for identifying novel objects and evaluating different candidate grasping strategies for an optimal grasping and handling which might be applied to the manufacturing of consumer electronic products. Learning methods may be used as well to perform the manipulation of flexible objects based on deformation models of elastic behaviours [39], enabling the system in real-time.

Advanced calibration methods allow a higher accuracy of micro-/nano-manipulation systems when working with vision-based systems. Xing et al. [40] propose an active calibration method to map from the relative motion of the multiple robot manipulators to the image coordination changes at the micro-scale, achieving high precision during assembly 
operations of irregular objects. The research also applies the method in positioning, tracking, and motion optimization on a micro-assembly platform. Shao et al. [41] introduce a calibration method for orientation alignment of components, including image coordinate system calibration, based on transfer matrix models. In [42], an efficient calibration method is presented which achieves high accuracy and low end-effector error for micro-manipulation and assembly tasks by computing the rototranslation matrix between the different systems.

\section{Autonomous Systems for Assembly Operations}

Autonomous behaviours help with complex assembly sequences in large ranges of motion while maintaining highaccuracy positioning requirements. Moreover, autonomous reconfigurable abilities may be achieved by implementing self-learning techniques and analyzing past assembly configurations, reaching higher effectiveness and efficiency during setup operations which are especially important to maximize throughput rates.

Assembly planning algorithms to perform autonomous assembly operations are based on shape recognition techniques of micro-objects. A matching algorithm was applied in [43], based on shape recognition for micro-assembly, allowing stability, high recognition efficiency ( $97 \%$ in $58.6 \mathrm{~ms}$ in average), and accurate positioning ( $93 \%$ in $20.1 \mathrm{~ms}$ in average) of the components. In [44], a sequential function chart was implemented in order to achieve automated procedures for micro-assembly, obtaining relatively more complex microassemblies by the system introduced lately by the same authors in [45]. This last work introduces a vision feedback strategy based on a real-time visual tracking algorithm used by the association of $2 \mathrm{D}$ features taken from images and their correspondence in the $\mathrm{CAD}$ model for the automation of assembly. A similar closed-loop control strategy is presented in [46] in which the 3D CAD is also used to present a complete description of the observed scene, including the effects of occlusion, for a 6-DOF tracking in real time. The works of Venkatesan et al. [47] and Venkatesan and Cappelleri [48] are based on sub-assembly sequence generation to find each optimal trajectory by a learned algorithm. Kim et al. [49] present a dexterous and fast micro-hand $(1 \mathrm{~mm} / \mathrm{s})$ system used for a high-speed $(100-\mu \mathrm{m}$ spheres in $800 \mathrm{~ms}$ and $1.54 \mathrm{~mm}$ in $13 \mathrm{~s})$ and precise assembly method at a large distance $(13 \mathrm{~mm})$ by implementing automatic releasing and error recovery system.

As an evolution to accomplish more working area, more DOF, or more stable assembly operations, a multi-mobile micro-manipulation system may be implemented. In [17], a multi-mobile micro-manipulation system is developed, which is capable of performing multiple robotic micro-assemblies by the cooperation of different manipulation systems, saving energy space and resources in industrial environments. Furthermore, in [50] a 16 magnetically driven milli-robot platform $(288 \times 288 \mathrm{~mm})$ has been developed to assemble carbon fibre rods, liquid adhesives, surface mount components (SMC), fibre glass sheets, and micro-components. In [51], a micro-robot-based micro-assembly sequence planning is presented by the optimization of assembly flexibility and reorientation numbers as the object function. The hybrid algorithm combines the idea of particle swarm (PS) optimization and ant colony optimization algorithms (ACO), requiring some previous offline, non-autonomous work.

\section{Introducing the Human Factor in Micro-/Nano- manufacturing}

Despite the continuous search for more autonomous systems that do not require human interventions, some specific tasks will still require a human-in-the-loop approach, for monitoring purposes or due to the human ability to adapt to unforeseen perturbations or the high complexity of assembly operations [21] [22] [23]. For that reason, human role in manufacturing gains importance as a more natural interaction is required to take advantage of both actors [8]. From an operational point of view, the last developments in HumanRobot Collaboration (HRC) strategies improved the active role of operators in automatic assembly operations performed by robots and normally applied at the macro-scale. Humanrobot interactions (HRI) may be classified as follows [52]: (i) coexistence, (ii) interaction, (iii) cooperation, and (iv) collaboration. Therefore, common control approaches are categorized in [52] [53] (a) tracking, understanding, and predicting real-time human motions in dynamic environments; (b) predictive and reactive strategies in collision detection for control adaptation and reaction; and (c) planning and adapting motions and tasks in real time.

To improve these interactions in complex environments, other approaches focus on developing cognitive abilities of these systems which consist in appropriate models and representations of the context surrounding the interaction [54]. Researches in this area focus on (i) cognition systems applied to robots (symbolic and spatial cognition); (ii) the interactions itself (dialog, action, intention, control and action planning models); and (iii) human partner cognition (mental models and social cognition). DT represents a perfect framework to orchestrate, execute the models, and analyze these collaborations by performing assembly simulations in a virtual environment [55].

VR devices are exploited for assembling complex hybrid MEMS devices by teleoperation [56] [57], for assembly planning [58] [59] and teaching purposes [33]. An important improvement of manufacturing industry is the development of technologies that enables a collaborative process involving distributed teams of engineers. In this context, authors in [60] outline the convenience of VR systems combined with web-based technologies, in which VR technologies enable 
engineers to study assembly sequences automatically generated for micro-assembly processes as an answer to a globally changing customer requirements of micro-devices. For a complete immersion of operators in the virtual environment, Chiou and Kwon [61] include a grip force collection for remote force feedback control, acting as a haptic system as developed in [62].

In order to achieve a complete collaboration between robot and humans, a high-speed visual feedback to recognize human movements is required. For this purpose, authors in [63] develop a collaborative micro-assembly peg-in-hole system with a 3 dof manipulator and an expandable tracking area method by composing the images taken from two cameras which achieves a precision of about $10 \mu \mathrm{m}$. To compensate operational errors of the VR system, Chang and Jau [64] present a calibration strategy that measures the error between representative points in the virtual world and the charge-couple device (CCD) visual system and corrects the projection matrix.

Finally, augmented reality (AR) and mixed reality (MR) systems may complement and compensate the insufficient information taken from physical vision-based manipulation systems and put it available to operators, as well. For example, in [65], an advanced MR system was used in manual or automated micro peg-in-hole operations, justifying an improvement in efficiency and effectiveness of the micro-assembly operations.

\section{Evolvable and Modular Micro-/Nano-assembly Systems and Control Strategies}

CPPS deployment requires both a physical and a computational infrastructure to support all aforementioned systems while achieving features as evolvability and adaptability, maintaining system performance as well. To accomplish these goals, different frameworks have been developed during the last decade, from a more physical deployment to advance control software architectures.

The concept of Evolvable Production Systems (EPS) [66] [67] stands for multi-product lines with reusable equipment for easy and fast changes of the physical modules, according to production demands. Following the mini/micro-factory paradigm and the EPS concept, authors in [68] highlight the closer link between the product design, the process sequences, manufacturing processes, and module used, presenting the Evolvable Micro Production System (EMPS) concept when considering the strict specificities of micro-product manufacturing. Some representative platforms are presented as well, as the TUT Microfactory [69] and the microFLEX [70].

On the other hand, information-centric approaches and frameworks enable a more efficient manufacturing and autonomous behaviours. In [18] and [71••], an advanced IoT-based CPS framework that uses cloud-based principles for micro- device assembly in distributed working locations is presented. These frameworks are capable of real-time data management to directly feed the simulations on the cyber counterpart of the CPPS allowing rapid data analysis and information availability.

Realistic simulations of the physical world for accurate manipulation are of great relevance, although very complex at the micro-scale. In this direction, in [72••], a DT-driven control framework is presented as a base to implement these simulations. The importance and the potential of digital-twin technologies to meet high standards of accuracy and dimensional performance required by assembly processes are highlighted. The work implements a DT framework based on the relationship between the physical and the virtual assembly space to improve the operational methods, defining different models (assembled part model, assembly process model, and assembly performance model) to perform and optimize the entire assembly process.

Nonetheless, smart manufacturing still requires decentralized control structures based on intelligent entities. As a solution, the concept of agents was introduced, representing fundamental processing units that build systems with cognitive capabilities. Early researches addressed the management and control of modular systems developing standardized methods and effective tools based on multi-agent architectures for precision assembly systems [73]. However, only few tackled the specific control requirements that microassembly systems present. Gendreau et al. [12] presented a method for designing a control architecture which allows the system to adapt its organization structure and its control strategy, based on two complementary approaches coming from functional analysis and technological constraints of microcomponents. A robotic station is presented which is able to teleoperate micro-assemblies and perform pick-and-place operations of objects sized around $40 \mu \mathrm{m}$. Another modular system was presented in [69] from the environmental, economic, and social perspectives.

\section{Conclusions}

This work analyzed the research trends in micro-/nano-assembly operations from the perspective of a Cyber-Physical Production Systems (CPPS) implementation. CPPS represents a powerful context for manufacturing to meet current market requirements by combining computational and physical tools and methods in different domains. However, a common framework and a holistic approach, including the sustainability asset, is still missing [74], thus limiting its robustness and industrial applicability, at all manufacturing levels.

Some advances have been achieved in the micro-/nanomanipulation domain showing the potential of CPPS approaches, especially in the context of distributed systems 
and VR environments by the realization of cyber-physical frameworks. They represent a solid foundation to continue developing more sophisticated and precise systems for micro/nano-manufacturing based on multi-agent-based control architectures, advanced algorithms, and realistic simulations. Smart features and self-x behaviours will face with the problem of evolvability in the form of autonomously reconfigurable and collaborative modules that allow an optimal micro/nano-production with higher throughput.

Lastly, being the role of human operators still crucial in this manufacturing field, it is interesting to develop solutions for cooperative and collaborative operations, i.e. for planning and/or assembly micro-/nano-operations. The intervention might be seen not only as a supervisor, but also as a collaborative workmate, exploiting human-robot interactions to take advantage of both the human dexterity and flexibility and the reliability and precision of the robots.

Funding This work has been developed in the context of the H2020MSCA-ITN DiManD project funded by the European Union's Horizon 2020 research and innovation programme under grant agreement no. 814078 .

\section{Compliance with Ethical Standards}

Conflict of Interest The authors declare that they have no conflict of interest.

Human and Animals Rights and Informed Consent This article does not contain any studies with human or animal subjects performed by any of the authors.

Open Access This article is licensed under a Creative Commons Attribution 4.0 International License, which permits use, sharing, adaptation, distribution and reproduction in any medium or format, as long as you give appropriate credit to the original author(s) and the source, provide a link to the Creative Commons licence, and indicate if changes were made. The images or other third party material in this article are included in the article's Creative Commons licence, unless indicated otherwise in a credit line to the material. If material is not included in the article's Creative Commons licence and your intended use is not permitted by statutory regulation or exceeds the permitted use, you will need to obtain permission directly from the copyright holder. To view a copy of this licence, visit http://creativecommons.org/licenses/by/4.0/.

\section{References}

Papers of particular interest, published recently, have been highlighted as:

- Of importance

-• Of major importance

1. Boudet J, Gregg B, Rathje K, Stein E, Vollhardt K McKinsey \& Company. The future of personalization - and how to get ready for it. [Online].; 2019 [cited 2020 September. Available from: https:// www.mckinsey.com/business-functions/marketing-and-sales/ourinsights/the-future-of-personalization-and-how-to-get-ready-for-it.

2. Zhou K, Liu T, Zhou L. Industry 4.0: towards future industrial opportunities and challenges. In: In 12th International Conference on Fuzzy Systems and Knowledge Discovery (FSKD). Zhangjiajie; 2015. https://doi.org/10.1109/FSKD.2015.7382284.

3. Zhong RY, Xu X, Klotz E, Newman ST. Intelligent manufacturing in the context of Industry 4.0: a review. Engineering. 2017;3(5): 616-30. https://doi.org/10.1016/J.ENG.2017.05.015.

4. Sanderson D, Chaplin JC, de Silva L, Holmes P, Ratchev S. Smart manufacturing and reconfigurable technologies: towards an integrated environment for evolvable assembly systems. In: In IEEE 1st International Workshops on Foundations and Applications of Self-* Systems. Augsburg; 2016. https://doi.org/10.1109/FAS-W. 2016.61.

5. Pfrommer J, Stogl D, Aleksandrov K, Navarro SE, Hein B, Beyerer J. Plug \& produce by modelling skills and service-oriented orchestration of reconfigurable manufacturing systems. AtAutomatisierungstechnik. 2015;63(10):790-800. https://doi.org/ 10.1515/auto-2014-1157.

6. Monostori L, Kádár B, Bauernhansl T, Kondoh S, Kumara S, Reinhart G, et al. Cyber-physical systems in manufacturing. CIRP Ann Manuf Technol. 2016;65:621-41. https://doi.org/10.1016/j. cirp.2016.06.005.

7. Monostori L. Cyber-physical production systems: roots, expectations and R\&D challenges. In: CIRP P, editor. editor 47th CIRP Conference on Manufacturing Systems, vol. 17. Ontario: Windsor; 2014. p. 9-13. https://doi.org/10.1016/j.procir.2014.03.115.

8. Patel R, Hedelind M, Lozan-Villegas P. Enabling robots in smallpart assembly lines: the "ROSETTA approach" - an industrial perspective. In: In ROBOTIK. Munich: 7th German Conference on Robotics; 2012. p. 279.

9. Hedelind M, Kock S. Requirements on flexible robot systems for small parts assembly: a case study. In: In IEEE International Symposium on Assembly and Manufacturing (ISAM). Tampere; 2011. https://doi.org/10.1109/ISAM.2011.5942356.

10. Kock S, Vittor T, Matthias B, Jerregard H, Kälman M, Lundberg I. Robot concept for scalable, flexible assembly automation: a technology study on a harmless dual-armed robot. In: In IEEE International Symposium on Assembly and Manufacturing (ISAM). Tampere; 2011. https://doi.org/10.1109/ISAM.2011. 5942358.

11. MarketsandMarkets. Industry 4.0 market by technology (IoT, artificial intelligence, industrial metrology, industrial robotics, AR \& VR, blockchain, 3D printing, digital twin, and 5G - offering, application, and end users) and geography- Global Forecast to 2024. ; 2019. https://www.marketsandmarkets.com/Market-Reports/ industry-4-market-102536746.html.

12. Gendreau D, Gauthier M, Hériban D, Lutz P. Modular architecture of the microfactories for automatic micro-assembly. Robotics and Computer-Intergrated Manufacturing. 2010;26:354-60. https://doi. org/10.1016/j.rcim.2009.11.013.

13. Papo DO, Stepjanou HE. Micro and mesoscale robotic assembly. J Manuf Process. 2004;6(1):-71. https://doi.org/10.1016/S15266125(04)70059-6.

14. Banerjee AG, Gupta SK. Research in automated planning and control for micromanipulation. IEEE Trans Autom Sci Eng. 2013;10, 10(3):-495. https://doi.org/10.1109/TASE.2013.2260539.

15. Dejeu J, Bechelany M, Rougeot P, Philippe L, Gauthier M. Adhesion control for micro- and nanomanipulation. ACS Nano. 2011;5(6):4648-57. https://doi.org/10.1021/nn200658z.

16. Gauthier M, Regnier S, Rougeot P, Chaillet N. Analysis of forces for micromanipulations in dry and liquid media. $\mathrm{J}$ Micromech. 2006;3(3-4):389-413. https://doi.org/10.1163/ 156856306777924699 . 
17. Jain RK, Majumder S, Ghosh B, Saha S. Analysis of multiple robotic assemblies by cooperation of multimobile micromanipulation systems (M4S). International journal of Adnvanced Manufacturing Technology. 2017;91:9-12. https://doi.org/10. 1007/s00170-016-9969-2.

18. Gunda R, Cecil J, Calyam P, Kak S. Information centric frameworks for micro assembly. In On the Move to Meaningful Internet Systems: Workshop. 2011. https://doi.org/10.1007/978-3642-25126-9 17.

19. Rasheed A, San O, Kvamsdal T. Digital twin: values, challenges and enablers from a modeling perspective. IEEE Access. 2020;8: 21980-2012. https://doi.org/10.1109/ACCESS.2020.2970143.

20. Grieves M, Vickers J. Digital twin: mitigating unpredictable, undesirable emergent behavios in complex systems. In Kahlen FJ, Flumerfelt S, Alves AC. Transdisciplinary perspectives on complex systems: new findings and approaches.:2017.85-113. https://doi. org/10.1007/978-3-319-38756-7_4

21. Dobra Z, Dhir KS. Technology jump in the industry: human-robot cooperation in production. Ind Robot. 2020;47(5):757-75.

22. Gajsek B, Stradovnik S, Hace A. Sustainable move towards flexible, robotic, human-involving workplace. Sustainability. 2020;12: 6590. https://doi.org/10.3390/su12166590.

23. Faccio M, Minto R, Rosati G, Bottin M. The influence of the product characteristics on human-robot collaboration: a model for the performance of collaborative robotic assembly. The International Journal of Adnvance Manufacturing Technology. 2019;106: 2317-31. https://doi.org/10.1007/s00170-019-04670-6.

24. Sisinni E, Saifullah A, Han S, Jennehag U, Gidlund M. Industrial internet of things: Challenges, opportunities, and directions. IEEE Transactions on Industrial Informatics. 2018;14:14(11)-4734. https://doi.org/10.1109/TII.2018.2852491.

25. Monostori L, Váncza J, Kumara SR. Agent-based systems for manufacturing. CIRP Ann Manuf Technol. 2006;55(2):697-720. https://doi.org/10.1016/j.cirp.2006.10.004.

26. Andreadis G, Klazaglou P, Niotaki K, Bouzakis KD. Classification and review of multi-agents systems in the manufacturing section. Procedia Engineering. 2014;69:282-90. https://doi.org/10.1016/j. proeng.2014.02.233.

27. Fontana G, Ruggeri S, Fassi I, Legnani G. A mini work-cell for handling and assembling microcomponents. Assem Autom. 2014;34(1):27-33. https://doi.org/10.1108/AA-11-2012-087.

28. Wei Y, Xu Q. An overview of micro-force sensing techniques. Sensors Actuators A Phys. 2015;123:359-74. https://doi.org/10. 1016/j.sna.2015.09.028

29. Cappelleri DJ, Adam G. Towards a real-time $3 \mathrm{~d}$ vision-based micro-force sensing probe. Journal of Micro-Bio Robotics. 2020;16: 23-32. https://doi.org/10.1007/s12213-019-00122-2.

30. Jing W, Chowdhury S, Guix M, Wang J, An Z, Johnson BV, et al. A microforce-sensing mobile microrobot for automated micromanipulation tasks. IEEE Trans Autom Sci Eng. 2019;16, 16(2):-530. https://doi.org/10.1109/TASE.2018.2833810.

31. Zhao Y, Huang X, Liu Y, Wang G, Hong K. Design and control of a piezoelectric-driven microgripper perceiving displacement and gripping force. Micromachines. 2020;11(2):121. https://doi.org/ 10.3390/mi1 1020121.

32. Jain RK, Majumder S, Ghosh B, Saha S. Design and manufacturing of mobile micro manipulation system with a compliant piezoelectric actuator based micro gripper. J Manuf Syst. 2015;35:76-91. https://doi.org/10.1016/j.jmsy.2014.12.001.

33. Hamdi M, Ferreira A. Microassembly planning using physicalbased models in virtual environment. In: In IEEE/RSJ International Conference on Intelligent Robotics and Systems. Sendai; 2004.

34. Wang $\mathrm{P}, \mathrm{Xu}$ Q. Design and modelling of constant-force mechanism: a survey. Mech Mach Theory. 2018;119:1-21. https://doi. org/10.1016/j.mechmachtheory.2017.08.017.
35. Kleeberger K, Bormann R, Kraus W, Huber MF. A survey on learning-based robotic grasping. Current Robotics Reports. 2020;1:239-49. https://doi.org/10.1007/s43154-020-00021-6.

36. Bafuma Liseli J, Dahmouche R, Kumar P, Seon JA, Gauthier M. Enhancing in-hand dexterous micro-manipulation for real-time applications. In: In International Conference on Automation Science and Engineering (CASE). Munich; 2018. https://doi.org/10.1109/ COASE.2018.8560362.

37. Seon JA, Dahmouche R, Gauthier M. Enhance in-hand dexterous micromanipulation by exploiting adhesion forces. IEEE Transactions on Robotics. 2018;34(1):113-25. https://doi.org/10. 1109/TRO.2017.2765668 The results of this work highlight the role of adhesion forces during the manipulation of microobjects and how advanced control systems may exploit these physical interactions for process optimization.

38. van Vuuren JJ, Tang L, Al-Bahadly I, Arif K. Towards the autonomous robotic gripping and handling of novel objects. In: In 14th IEEE Conference on Industrial Electronics and Applications (ICIEA). Xi'an; 2019. https://doi.org/10.1109/ICIEA.2019. 8833640.

39. Bodenhagen L, Fugl AR, Willatzen M, Petersen HG, Krüger N. Learning peg-in-hole actions with flexible objects. In: In 4th International Conference on Agents and Artificial Intelligence (ICAART). Vilamoura; 2012.

40. Xing D, Xu D, Li H, Luo L. Active calibration and its applications on micro-operating platform with multiple manipulators. In: In IEEE International Conference on Robotics \& Automation (ICRA). Hong Kong; 2014. https://doi.org/10.1109/ICRA.2014. 6907661.

41. Shao C, Ye X, Qian J, Zhang Z, Zhu D. Robotic precision assembly system for microstructures. Journal of Systems and Control Engineering. 2020;234(8):948-58. https://doi.org/10.1177/ 0959651819885755.

42. Fontana G, Ruggeri S, Legnani G, Fassi I. Unconventional calibration strategies for micromanipulation work-cells. Robotica. 2018;36(12):1897-919. https://doi.org/10.1017/ S0263574718000796.

43. Zhang JY, Xu SP, Liu Y, Hao YP. Research on the identification method of micro assembly part. In: In 2nd Conference on Image, Vision and Computing (ICIVC). Chengdu; 2017. https://doi.org/10. 1109/ICIVC.2017.7984564.

44. Tamadazte B, Le Fort-Piat N, Dembélé S, Fortier G. Robotic micromanipulation for microassembly: modelling by sequential function chart and achievement by multiple scale visual servoings. Journal of Micro-Nano Mechatronics. 2009;5:1-12.

45. Brahim T, Marchand E, Dembélé S, Le For-Piat N. CAD modelbased tracking and 3D visual-based control for MEMS microassembly. The International Journal of Robotics Research. $2010 ; 11(29): 1416-34$. https://doi.org/10.1177/ 0278364910376033 .

46. Yesin KB, Nelson BJ. A CAD model based tracking system for visually guided microassembly. Robotica. 2005;23:409-18. https:// doi.org/10.1017/S0263574704000840.

47. Venkatesan V, Seymour J, Cappelleri DJ. Microassembly sequence and path planning using sub-assemblies. Journal of Mechanisms and Robotics. 2018;10(6). https://doi.org/10.1115/1.4041333.

48. Venkatesan V, Cappelleri DJ. Path planning and micromanipulation using a learned model. IEEE Robotics and Automation Letters. 2018;3(4):3096. https://doi.org/10.1109/LRA.2018.2849568.

49. Kim E, Kojima M, Mae Y, Arai T. High-speed manipulation of microobjects using an automated two-fingered microhand for $3 \mathrm{~d}$ microassembly. Micromachines. 2020;11:534. https://doi.org/10. 3390/mi11050534.

50. Hsu A, Zhao H, Gaudreault M, Foy AW, Pelrine R. Magnetic millirobot swarm platform: a safety barrier certificate enabled, low-cost 
test bed. IEEE Robotics and Automation Letters. 2020;5(2):2920. https://doi.org/10.1109/LRA.2020.2974713.

51. Shuang B, Chen J, Li Z. Microrobot based micro-assembly sequence planning with hybrid and colony algorithm. Internation Journal on Manufacturing Technology. 2008;38:1227-35. https:// doi.org/10.1007/s00170-007-1165-y.

52. Wang L, Gao RX, Váncza J, Krüger J, Wang XV, Makris S, et al. Symbiotic human-robot collaborative assembly. CIRP Ann. 2019;68(2):701-26. https://doi.org/10.1016/j.cirp.2019.05.002.

53. Haddadin S, Croft E. Physical human-robot interaction. In Siciliano B, Khatib O. Springer handbook of robotics.:2016.1835-1874. https://doi.org/10.1007/978-3-319-32552-1_69.

54. Mutlu B, Roy N, Sabanovic S. Cognitive human-robot interaction. In Siciliano B, Khatib O. Springer Handbook of Robotics.: 2016.1907-1934. https://doi.org/10.1007/978-3-319-32552-1 71.

55. Bilberg A, Malik AA. Digital twin driven human-robot collaborative assembly. CIRP Ann Manuf Technol. 2019;68:499-502. https://doi.org/10.1016/j.cirp.2019.04.011.

56. Ammi M, Ferreira A, Fontaine JG. Virtualized reality interface for tele-micromanipulation. In: In IEEE International Conference on Robotics \& Automation (ICRA). New Orleans; 2004. https://doi. org/10.1109/robot.2004.1307481.

57. Probst M, Hürzeler C, Borer R, Nelson BJ. Virtual Reality for microassembly. In Proceedings of SPIE - The International Society for Optical Engineering. 2007. https://doi.org/10.1117/12. 754557.

58. Cassier C, Ferreira A, Hirai S. Combination of vision servoing techniques and VR-based simulation for semi-autonomous microassembly workstation. In: In IEEE International Conference on Robotics \& Automation (ICRA). Washington, D.C.; 2002. p. 1501-6. https://doi.org/10.1109/ROBOT.2002.1014756.

59. Cecil J, Huber J, Gobinath N, Jacques J. A virtual factory environment to support process design in micro assembly domains. Computer-Aided Design \& Applications. 2011;8(1):119-27. https://doi.org/10.3722/cadaps.2011.119-127.

60. Cecil J, Jones J. VREM: An advanced virtual environment for micro assembly. Int J Adv Manuf Technol. 2014;72(1-4):47-56. https://doi.org/10.1007/s00170-014-5618-9.

61. Chiou R, Kwon Y. Remotely adjustable robotic grip force for the network-based assembly automation. Internation Journal on Advance Manufacturing technology. 2011;54:1145-54. https:// doi.org/10.1007/s00170-010-2979-6.

62. Mehrtash M, Khamesee MB, Tarao S, Tsuda N, Chang JY. Humanassisted virtual reality for a magnetic-haptic micromanipulation platform. Microsystems Technology. 2012;18(9-10):1407-15. https://doi.org/10.1007/s00542-012-1560-7.

63. Kojima O, Huang S, Marakami K, Ishikawa M, Yamakawa Y. Human-robot interaction system for micromanipulation assistance. In: In IECON - 44th Annual Conference of the IEEE Industrial Electronics Society. Washington D.C.; 2018. https://doi.org/10. 1109/IECON.2018.8592819.

64. Chang RJ, Jau JC. Error measurement and calibration in developing virtual-reality-assisted microassembly system. International Journal of Automation Technology. 2015;9(6). https://doi.org/10.20965/ ijat.2015.p0619.

65. Chang RJ, Jau JC. Augmented reality in peg-in-hole microassembly operation. International Journal of Automation Technology. 2016;10(3):438-46. https://doi.org/10.20965/ijat. 2016.p0438.

66. Onori M, Barata J, Frei R. Evolvable assembly systems: basic principles. International Federation for Information Processing (IFIP). 2006;220:317-28.

67. Onori M, Semere D, Lindberg B. Evolvable systems: an approach to self-X production. Int J Comput Integr Manuf. 2011;24(5):50616.

68. Hofmann A, Bretthauer G, Siltala N, Tuokko R. Evolvable Micro Production Systems: specific needs and differences to macro. In: In IEEE International Symposium on Assembly and Manufacturing (ISAM). Tampere; 2011. p. 1-6. https://doi.org/10.1109/ISAM. 2011.5942361.

69. Järvenpää E, Heikkilä R, Tuokko R. TUT-microfactory - a smallsize, modular and sustainable production. In: In 11th Global Conference on Sustainable Manufacturing. Berlin; 2013.

70. Hofmann A, Hummel B, Firat O, Bretthauer G, Bär M, Meyer M. microFLEX - a new concept to address the needs for adaptable meso and micro assembly lines. In: In IEEE International Symposium on Assembly and Manufacturing (ISAM). Tampere; 2011. https://doi.org/10.1109/ISAM.2011.5942297.

71.• Cecil J, Albuhamood S, Cecil-Xavier A, Ramanathan P. An advanced cyber physical framework for micro devices assembly. IEEE Transactions on Systems Manufacturing and Cybernetics. 2019;49(1):92-106. https://doi.org/10.1109/TSMC.2017.2733542 This work discusses a major advance in manufacturing by the deployment of a collaborative demonstrator and shows the potential of CPS for micro/nano-manufacturing assembly operations.

72.• Xuemin S, Jinsong B, Yiming ZS, Bin Z. A digital twin-driven approach for the assembly-commissioning of high precision products. Robotics and Computer Integrated Manufacturing. 2020:61. https://doi.org/10.1016/j.rcim.2019.101839 This complete work represents a state-of-the-art example of how CPS can be implemented and how this framework allows the analysis and optimization of the entire assembly process.

73. Ferreira P, Lohse N, Ratchev S. Multi-agent architecture for reconfiguration of precision modular assembly systems. In: In International Precision Assembly Seminar (IPAS). Chamonix; 2010. https://doi.org/10.1007/978-3-642-11598-1_29.

74. Weyer S, Schmitt M, Ohmer M, Gorecky D. Towards Industry 4.0 standardization as the cruzial challenge for highly modular, multivendor production systems. 15th IFAC Symposium on Information Control Problems in Manufacturing (INCOM). 2015;48(3):579-1.

Publisher's Note Springer Nature remains neutral with regard to jurisdictional claims in published maps and institutional affiliations. 